sciendo

\title{
WORD METRIC, STATIONARY MEASURE AND MINKOWSKI'S QUESTION MARK FUNCTION
}

\author{
URIYA PUMERANTZ
}

\author{
Department of Mathematics, Tel Aviv University, Tel-Aviv, ISRAEL
}

\begin{abstract}
Given a countably infinite group $G$ acting on some space $X$, an increasing family of finite subsets $G_{n}, x \in X$ and a function $f$ over $X$ we consider the sums $S_{n}(f, x)=\sum_{g \in G_{n}} f(g x)$. The asymptotic behaviour of $S_{n}(f, x)$ is a delicate problem that was studied under various settings. In the following paper we study this problem when $G$ is a specific lattice in SL $(2, \mathbb{Z})$ acting on the projective line and $G_{n}$ are chosen using the word metric. The asymptotic distribution is calculated and shown to be tightly connected to Minkowski's question mark function. We proceed to show that the limit distribution is stationary with respect to a random walk on $G$ defined by a specific measure $\mu$. We further prove a stronger result stating that the asymptotic distribution is the limit point for any probability measure over $X$ pushed forward by the convolution power $\mu^{* n}$.
\end{abstract}

\section{Communicated by Oleg Karpenkov}

\section{Introduction}

Let $G$ be a countably infinite group acting on some space $X$. A natural question to study is the asymptotic behaviour of orbits when the elements are chosen using different laws. More precisely, we ask if for a fixed filtration into finite subsets $G_{t} \nearrow G$ and a function $f$ over $X$ there exists a normalization function $c(t): \mathbb{R}^{+} \rightarrow \mathbb{R}$ such that the limit

$$
\lim _{t \rightarrow \infty} \frac{1}{c(t)} \sum_{g \in G_{t}} f(g x) \quad \text { exists . }
$$

(C) 2020 BOKU-University of Natural Resources and Life Sciences and Mathematical Institute, Slovak Academy of Sciences.

2010 Mathematics Subject Classification: 22E40, 30B70, 60G10.

Keywords: Stationary measure, Minkowski's question matk function, Word metric, Lattice orbits.

The support of ISF grant 2919/19 is gratefully acknowledged.

Licensed under the Creative Commons Attribution-NC-ND 4.0 International Public License. 
This subject was studied extensively in various settings, see e.g., in [7]-10]. However, none of these works explored the notion of choosing $G_{t}$ using the word metric, and little is known in this case. Through this paper we study this problem in a very specific settings. We choose $G$ to be the Farey group which is a well studied subgroup of PSL $(2, \mathbb{Z})$, endowed with the natural action on the projective line, and set carefully chosen generators that allow reaching a solution with interesting properties.

Definition 1.1. The subgroup of PSL $(2, \mathbb{Z})$ generated by

$$
a=\left[\left(\begin{array}{ll}
1 & -2 \\
1 & -1
\end{array}\right)\right], \quad b=\left[\left(\begin{array}{rr}
0 & -1 \\
1 & 0
\end{array}\right)\right], \quad c=\left[\left(\begin{array}{ll}
1 & -1 \\
2 & -1
\end{array}\right)\right]
$$

is called the Farey group.

Theorem 1.1. Let $\Gamma$ be the Farey group. Let $\|\cdot\|$ denote the word metric with respect to $\{a, b, c\}$ and set $\Gamma_{n}=\{\gamma \in \Gamma:\|\gamma\|=n\}$. For $x \in X=P\left(\mathbb{R}^{2}\right)$, the projective line, $f: X \rightarrow \mathbb{R}, n \in \mathbb{N}$ we define

$$
S_{n}(f, x)=\sum_{\gamma \in \Gamma_{n}} f(\gamma x) .
$$

Then there exists a measure $\mu_{\overline{\mathcal{M}}}$ on $X$ such that for every $x \in X$ and every continuous $f$,

$$
\lim _{n \rightarrow \infty} \frac{S_{n}(f, x)}{\left|\Gamma_{n}\right|}=\int_{X} f \mathrm{~d} \mu_{\overline{\mathcal{M}}} .
$$

The measure $d \mu_{\overline{\mathcal{M}}}$, named the extended Minkowski measure, is constructed in the first section. For a natural parameterization of the projective line by $\mathbb{R} \cup\{\infty\}$, the cumulative distribution function of $d \mu_{\overline{\mathcal{M}}}$ is tightly connected to the Minkowski question mark function [1. This function, described in Section 2.3, is usually expressed in terms of continued fractions and is studied in the field of Diophantine approximations. We proceed to show that the extended Minkowski measure is, in fact, stationary with respect to a specific random walk on $X$.

TheOREM 1.2. The extend Minkowski measure $\mu_{\overline{\mathcal{M}}}$ is stationary with respect to the random walk generated by $\mu(a)=\mu(b)=\mu(c)=\frac{1}{3}$.

Stationary measures have great importance in dynamics. Particularly relevant to this paper are results by Furstenberg [3] showing existence and uniqueness of stationary measure for certain random walks on projective spaces. While there is a general result guaranteeing the existence of such a measure, it is rare to be able to explicitly express one. In the particular setting studied here, using unique 
properties of the Farey group, the stationary measure is not only explicitly expressed, but also shown to have a connection to a function from a seemingly unrelated area. Notice that there are no $\Gamma$ invariant measures on $X$, thus the extended Minkowski measure is stationary but not invariant with respect to $\Gamma$.

Lastly, we show general conditions on a random walk under which the word metric limit and the stationary measure coincide. As a direct consequence of the proof we see that the extended Minkowski measure is also the limit measure of any probability measure on $X$ pushed forward by the nth convolution power

$$
\mu^{* n}:=\underbrace{\mu * \mu * \cdots * \mu}_{\mathrm{n} \text { times }} .
$$

THEOREM 1.3. Under same settings as Theorem 1.2, for any probability measure $\pi$ on $X$, the following limit exists in the weak-* topology

$$
\lim _{n \rightarrow \infty} \mu^{* n} * \pi=\mu_{\overline{\mathcal{M}}}
$$

Notice that for any random walk defined by a measure $\nu$ on a compact space with unique stationary measure, the Cesàro limit $\frac{1}{n} \sum_{k=1}^{n} \nu^{* k} * \pi$ converges in weak-* topology to the stationary measure. However, the existence of a Cesáro does not imply Theorem 1.3 .

Acknowledgements. This paper is a part of the author's M.Sc. thesis at Tel Aviv University. It was conducted under the supervision of Barak Weiss, to whom I wish to extend my gratitude for a most resourceful guidance. The support of ISF grant 2919/19 is gratefully acknowledged.

\section{Preliminaries}

\subsection{Farey group and tessellation}

We first describe a construction of the hyperbolic Farey tessellation $\mathscr{T}$. For $\frac{p_{1}}{q_{1}}, \frac{p_{2}}{q_{2}} \in \mathbb{Q}$ with $\operatorname{gcd}\left(p_{i}, q_{i}\right)=1$ we define $\frac{p_{1}}{q_{1}} \oplus \frac{p_{2}}{q_{2}}=\frac{p_{1}+p_{2}}{q_{1}+q_{2}}$. The ordered Farey sequences $\mathscr{F}_{n}=\left(s_{1}^{n}, s_{2}^{n}, \ldots, s_{k(n)}^{n}\right)$ are then constructed using the recurrence relation

$$
\begin{gathered}
\mathscr{F}_{0}=(0,1)=\left(s_{1}^{0}, s_{2}^{0}\right), \\
\mathscr{F}_{n+1}=\left(s_{1}^{n}, s_{1}^{n} \oplus s_{2}^{n}, s_{2}^{n}, s_{2}^{n} \oplus s_{3}^{n}, s_{3}, \ldots, s_{k(n)-1}^{n} \oplus s_{k(n)}^{n}, s_{k(n)}^{n}\right) .
\end{gathered}
$$

The following lemma summarizes useful well known facts regarding the Farey sequences [1]. 


\section{LEMMA 2.1.}

(1) $\cup_{n=1}^{\infty} \mathscr{F}_{n}=\mathbb{Q} \cap[0,1]$.

(2) Every $q \in \mathbb{Q} \cap[0,1]$ appears no more than once in any $\mathscr{F}_{n}$.

Since for any Farey pair $p<q$, the inequality $p<p \oplus q<q$ holds, the ordered sequences $\mathscr{F}_{n}$ are in fact ordered using the usual order on the real line.

Definition 2.1. Two rationals $p, q \in \mathbb{Q}$ with $p<q$ are called a Farey pair if they are successive terms in some $\mathscr{F}_{n}$. That is, if exists $n \in \mathbb{N}$ and $i \in \mathbb{N}$ such that $p=s_{i}^{n}$ and $q=s_{i+1}^{n}$.

Let $\mathbb{H}:=\{z \in \mathbb{C}: \operatorname{Im}(z)>0\}$ be the upper complex half plane equipped with the hyperbolic metric. For a detailed description of the hyperbolic upper half plane model see [5]. For any two points in the boundary $s, t \in \partial \mathbb{H}=\mathbb{R} \cup\{\infty\}$, $s \neq t$ we denote by $l(s, t) \subset \mathbb{H}$ the unique infinite hyperbolic geodesic in $\mathbb{C}$ that have $\{s, t\}$ in his closure and define

$$
\mathscr{T}_{0}=\bigcup_{\substack{(p, q) \\ \text { Farey pair }}} l(p, q) \cup l(0, \infty) \cup(1, \infty),
$$

where the union is over all Farey pairs $(p, q) . \mathscr{T}_{0}$ is a set of boundary curves of a tessellation by ideal hyperbolic triangles of the region $\{z \in \mathbb{H}: 0 \leq R e(z) \leq 1\}$. To complete this to a tessellation of the entire hyperbolic plane we define $\tilde{\mathscr{T}}$ using integral translations of $\mathscr{T}_{0}$,

$$
\tilde{\mathscr{T}}=\bigcup_{n \in \mathbb{Z}}\left(n+\mathscr{T}_{0}\right)
$$

One can check that $\tilde{\mathscr{T}}$ is a set of boundary curves of a tessellation of $\mathbb{H}$. This tessellation is called the Farey tessellation. We denote $T(p, q, r):=l(p, q) \cup$ $l(q, r) \cup l(p, r)$ the ideal hyperbolic triangle with vertices at $-\infty<p<q<$ $r \leq \infty$. We refer to any ideal hyperbolic triangle $T(p, q, r) \subset \tilde{\mathscr{T}}$ as a "Farey tile" or simply as a "tile". We denote by $\mathscr{T}$ the set of all Farey tiles. Set $\Delta_{e}=$ $T(0,1, \infty) \in \mathscr{T}$, and let $\{a, b, c\}$ be the set of hyperbolic reflections described in Definition 1.1. One can check that $\{a, b, c\}$ are hyperbolic reflections on the edges of $\Delta_{e}$. By Poincaré's Theorem [6], the representation of the Farey group $\Gamma$ in terms of generators and relations is $\left\langle a, b, c \mid a^{2}=b^{2}=c^{2}\right\rangle$. The following lemma states that $\tilde{\mathscr{T}}$ can be described both in terms of Farey sequences and as $\Gamma$ orbit of $\Delta_{e}$.

LEMMA 2.2.

$$
\tilde{\mathscr{T}}=\Gamma \Delta_{e}=\bigcup_{\gamma \in \Gamma} \gamma \Delta_{e}
$$

Proof for the above lemma can be found in [2]. As in any discrete subgroup of isometries, there is a bijection between $\mathscr{T}$ and $\Gamma$ given by $g \rightarrow \Delta_{g}:=g \Delta_{e}$. 
Definition 2.2. Two tiles $\Delta_{g}, \Delta_{h} \in \mathscr{T}$ are called neighbours if they share a common edge, that is $\Delta_{g} \cap \Delta_{h}=l(s, t)$ for some $s, t \in \partial \mathbb{H}$.

LEMMA 2.3. $\Delta_{g}$ is a neighbour of $\Delta_{h}$ if and only if $h=g s$ with $s \in\{a, b, c\}$.

Pro of. If $h=g s$, then since $\Delta_{s}$ is a neighbour of $\Delta_{e}$ and since isometries move geodesics to geodesics, it follows that $\Delta_{h}=\Delta_{g s}=g s \Delta_{e}=g \Delta_{s}$ is a neighbour of $\Delta_{g}=g \Delta_{e}$. As each tile has exactly 3 neighbours and there are exactly 3 generators, the condition is necessary.

\subsection{Structure of the Farey tessellation}

In this section we prove some results regarding the structure of the group $\Gamma$.

Definition 2.3. Let $G$ be a group generated by a set $S \subset G$. Define the word metric on $G$ as follows: for any $g \neq e$ by $\|g\|_{S}=\min \left(\left\{n \mid g=s_{1} s_{2} \ldots s_{n}, s_{i} \in S\right\}\right)$ and $\|e\|_{S}=0$.

Throughout this paper, we omit the subscript $S$ when the generating set is implied. The following general lemma is a direct consequence of Corollary 1.4.8 in [4].

LEMMA 2.4. Let $G$ be a group defined in term of generators and relations by $<a_{1}, \ldots, a_{n} \mid a_{i}^{2}=e>$. Then each $g \in G$ has a unique representation $g=s_{1} \ldots s_{n}$ with $m=\|g\|$ and $s_{i} \in\left\{a_{1}, \ldots, a_{n}\right\}$. This representation has $s_{i} \neq s_{i+1}$ for $1 \leq i<m$.

One can see that $\Gamma$ satisfies the assumptions in Lemma 2.4.

Definition 2.4. A representation that satisfies the conditions of Lemma 2.4 is called a reduced representation.

The following lemmas summarize some important observations regarding the Farey tessellation.

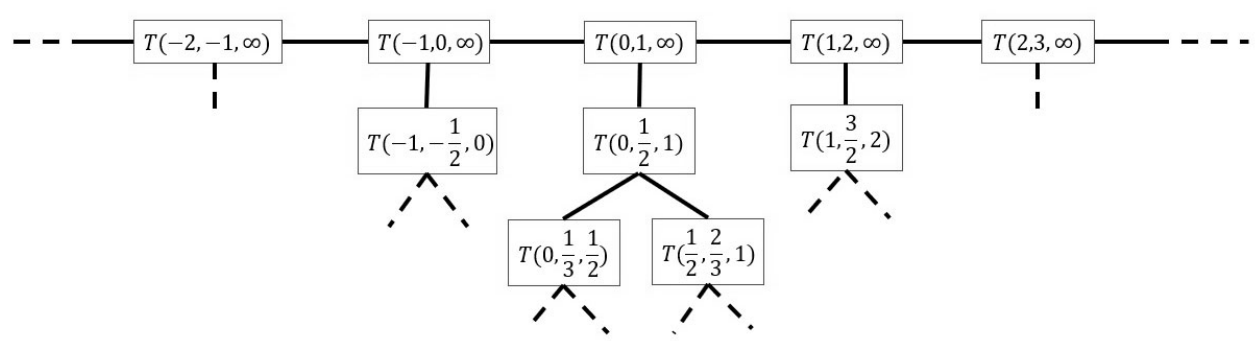

FiguRE 1. An illustration of the Farey tessellation structure. 
Lemma 2.5. Let $g \in \Gamma, g \neq e$ with a reduced representation $s_{1} \ldots s_{n}$ and let $s \in\{a, b, c\}$ such that $s \neq s_{n}$. Then:

(1) If $\Delta_{g}=T(m, m+1, \infty)$ and $m>0$, then $\Delta_{g s_{n}}=T(m-1, m, \infty)$ and either $\Delta_{g s}=T(m+1, m+2, \infty)$ or $\Delta_{g s}=T(m, m \oplus(m+1), m+1)$.

(2) If $\Delta_{g}=T(m, m+1, \infty)$ and $m<0$, then $\Delta_{g s_{n}}=T(m+1, m+2, \infty)$ and either $\Delta_{g s}=T(m-1, m, \infty)$ or $\Delta_{g s}=T(m, m \oplus(m+1), m+1)$.

(3) If $\Delta_{g}=T\left(q_{1}, q_{2}, q_{3}\right)$, then either $\Delta_{g s_{n}}=T\left(q_{1}, q_{3}, r\right)$ or $\Delta_{g s_{n}}=T\left(r, q_{1}, q_{3}\right)$ or $\Delta_{g s_{n}}=T\left(q_{1}, q_{3}, \infty\right)$ with $r \in \mathbb{Q}$ such that $q_{3}=q_{1} \oplus r$ or $q_{1}=r \oplus q_{3}$, respectively, and either $\Delta_{g s}=T\left(q_{1}, q_{1} \oplus q_{2}, q_{2}\right)$ or $\Delta_{g s}=T\left(q_{2}, q_{2} \oplus q_{3}, q_{3}\right)$.

Notice that for every $g \in \Gamma$ either $\Delta_{g}=(m, m+1, \infty)$ or $\Delta_{g}=T\left(q_{1}, q_{2}, q_{3}\right)$ with $m \in \mathbb{Z}$ and $q_{i} \in \mathbb{Q}$. The case $m=0$ has $\Delta_{g}=\Delta_{e}$, which makes it trivial.

P r o of. We will show a proof for the third case only. The other two cases are proved using identical reasoning. It follows from Lemma 2.3 that the neighbours of $\Delta_{g}$ are $\Delta_{g a}, \Delta_{g b}, \Delta_{g c}$. The construction of the Farey sequences, as described in terms of Farey sequences, implies that those tiles correspond to $T\left(q_{1}, q_{1} \oplus\right.$ $\left.q_{2}, q_{2}\right), T\left(q_{2}, q_{2} \oplus q_{3}, q_{3}\right)$ and either $T\left(q_{1}, q_{3}, r\right)$ or $T\left(r, q_{1}, q_{3}\right)$ or $T\left(q_{1}, q_{3}, \infty\right)$ with $r \in \mathbb{Q}$ such that $q_{3}=q_{1} \oplus r$ or $q_{1}=r \oplus q_{3}$, respectively. Assume by contradiction

$$
T_{g s_{n}}=T\left(q_{1}, q_{1} \oplus q_{2}, q_{2}\right):=T\left(q_{1}^{1}, q_{2}^{1}, q_{3}^{1}\right) .
$$

Then, since $s_{n} \neq s_{n-1}$ either

$$
T_{g s_{n} s_{n-1}}=T\left(q_{1}, q_{1} \oplus\left(q_{1} \oplus q_{2}\right), q_{1} \oplus q_{2}\right):=T\left(q_{1}^{2}, q_{2}^{2}, q_{3}^{2}\right)
$$

or

$$
T_{g g_{n} g_{n-1}}=T\left(q_{1} \oplus q_{2},\left(q_{1} \oplus q_{2}\right) \oplus q_{2}, q_{2}\right):=T\left(q_{1}^{2}, q_{2}^{2}, q_{3}^{2}\right) .
$$

Notice that

$$
\left\|g s_{n}\right\|=\|g\|-1
$$

In a similar way we may keep shortening $g$ until reaching $\Delta_{g s_{n} \ldots s_{1}}=\Delta_{e}=$ $T(0,1, \infty)=T\left(q_{1}^{n}, q_{2}^{n}, q_{3}^{n}\right)$. The intervals $\left[q_{1}^{i}, q_{3}^{i}\right]$ form a descending filtration and thus $1-0=q_{3}^{n}-q_{1}^{n}<q_{3}-q_{1}<1$, arriving at a contradiction. By same method we see $T_{g s_{n}} \neq T\left(q_{2}, q_{2} \oplus q_{3}, q_{3}\right)$, so the only possibility is that $\Delta_{g s_{n}}$ is either $T\left(q_{1}, q_{3}, r\right), T\left(r, q_{1}, q_{3}\right)$ or $T\left(q_{1}, q_{3}, \infty\right)$. The rest of the claim regarding the two other generators follows immediately.

LEMMA 2.6. Let $g, h \in \Gamma$ with reduced representations $g=g_{n} \ldots g_{1}, h=h_{m} \ldots h_{1}$ and $n \leq m$. Denote $\Delta_{g}=T\left(p_{1}, p_{2}, p_{3}\right)$ and $\Delta_{h}=T\left(q_{1}, q_{2}, q_{3}\right)$ with $p_{3}, q_{3}<\infty$. Then $\left[q_{1}, q_{3}\right] \subset\left[p_{1}, p_{3}\right]$ if and only if $h_{i}=g_{i}$ for all $1 \leq i \leq n$. 


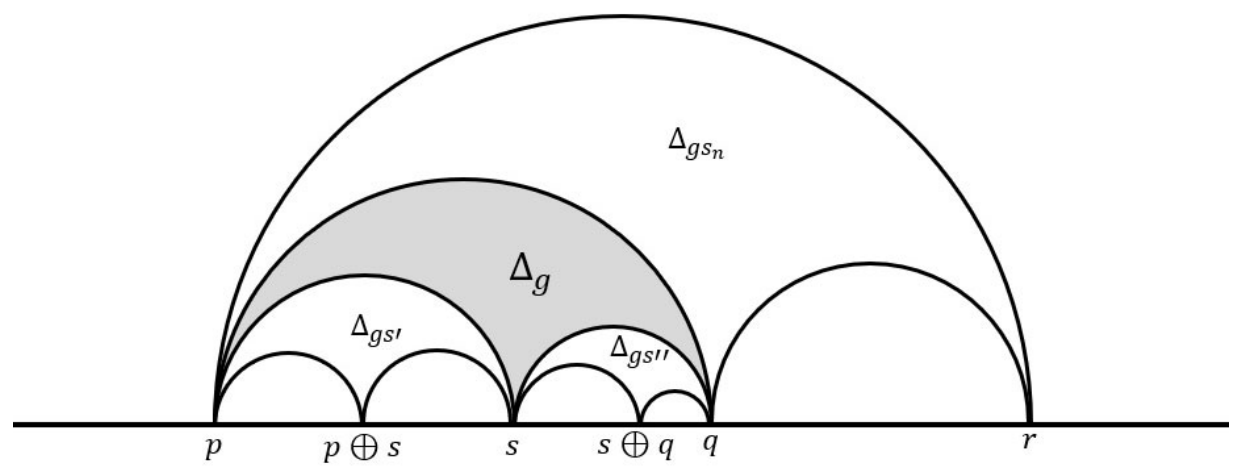

Figure 2. A tile with finite vertices.

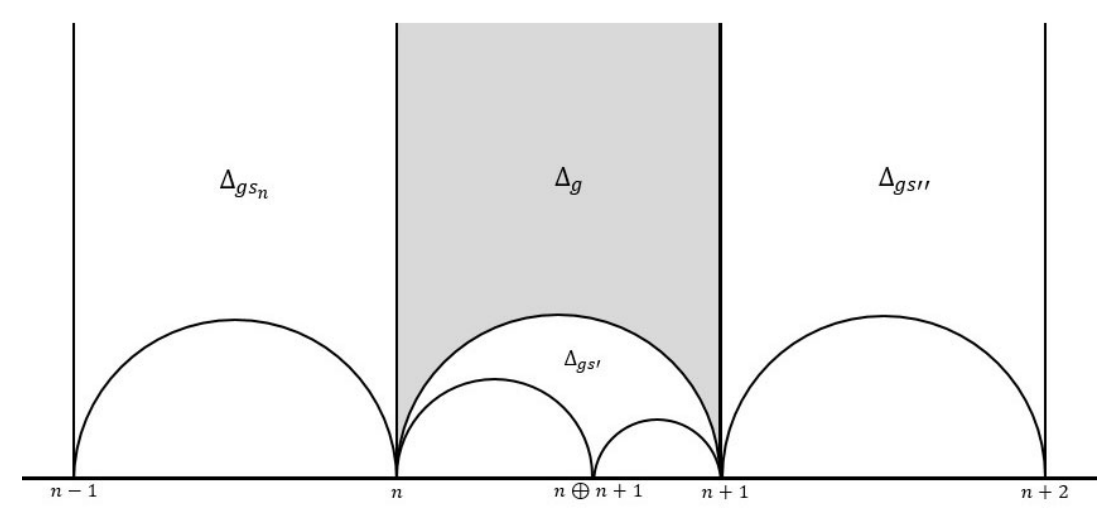

Figure 3. A tile with vertex at $\infty$.

Pr o of. Assume $h_{i}=g_{i}$ for all $1 \leq i \leq n$. Then

$$
\Delta_{h}=h_{m} \ldots h_{n+1} h_{n} \ldots h_{1} \Delta_{e}=h_{m} \ldots h_{n+1} g \Delta_{e}=h_{m} \ldots h_{n+1} \Delta_{g} .
$$

Since $h_{i} \neq h_{i+1}$ for all $i$ the claim follows from Lemma 2.5.

Let $2 \leq i_{0} \leq n$ be the first integer such that $h_{i_{0}} \neq g_{i_{0}}$. Then

$$
h_{i_{0}-1} \ldots h_{1} \Delta_{e}=g_{i_{0}-1} \ldots g_{1} \Delta_{e}=T\left(r_{1}, r_{2}, r_{3}\right) .
$$

For simplicity assume $r_{i}<\infty$, then since $h_{i_{0}} \neq g_{i_{0}}$ and both are different from $g_{i_{0}-1}=h_{i_{0}-1}$ we get by Lemma 2.5 that $g_{i_{0}} \ldots g_{1} \Delta_{e}=T\left(r_{1}, r_{1} \oplus r_{2}, r_{2}\right)$ and 
$h_{i_{0}} \ldots h_{1} \Delta_{e}=T\left(r_{2}, r_{2} \oplus r_{3}, r_{3}\right)$. Since $h, g$ are both given as reduced representation Lemma 2.5 finishes the proof. The other cases where $r_{3}=\infty$ are proven using Lemma 2.5 in a similar manner. The case $i_{0}=1$ is trivial.

The next lemma gives a characterization of some Farey tiles in terms of Farey sequences.

Lemma 2.7. Let $g \in \Gamma$ such that $\|g\|=n>0$ and $\Delta_{g}=T(p, r, q)$ with $0 \leq p<r<q \leq 1$. Then $r$ first appears in $\mathscr{F}_{n}$ and $p, q$ are a Farey pair in $\mathscr{F}_{n-1}$.

Pr o of. We prove by induction on $\|g\|$. Let $g=s_{1} \ldots s_{n}$ be the reduced representation of $g$. By Lemma 2.5 for $s \neq s_{n}, \Delta_{g s}=T(p, p \oplus r, r)$ or $\Delta_{g s}=$ $T(r, r \oplus q, q)$. Lemma 2.1 implies that both $p \oplus r$ and $r \oplus q$ first appear in $\mathscr{F}_{n+1}$. For the base case $\|g\|=1$, since $0 \leq p, r, q \leq 1$ it follows that $\Delta_{g}=T\left(0, \frac{1}{2}, 1\right)$. Since $\frac{1}{2} \in \mathscr{F}_{1}$ and $0,1 \in \mathscr{F}_{0}$ we are done.

The next lemma extends Lemma 2.7 to all tiles.

Lemma 2.8. Let $g \in \Gamma$ with $\|g\|=n>0$ and $\Delta_{g}=T(p, r, q)$. Then either $p=n, r=n+1, q=\infty$ or $p=-n, r=1-n, q=\infty$ or exists $m \in \mathbb{Z}$ such that $p-m, q-m$ are Farey pair in $\mathscr{F}_{n-m-1}$ and $r-m=(p-m) \oplus(q-m)$.

The proof is left as excercise for the reader.

Lemma 2.9. Let $\Gamma_{n}:=\{\gamma \in \Gamma:\|\gamma\|=n\}$. For $n \in \mathbb{N}, n>0$ :

$$
\left|\Gamma_{n}\right|=3 \cdot 2^{n-1} \text { and }\left|\Gamma_{0}\right|=1 \text {. }
$$

$\operatorname{Pr}$ o o f. For $n=0, \gamma=e$ is the only possible word hence $\left|\Gamma_{0}\right|=1$. For any $n \geq 1$ we use Lemma 2.4 and count reduced representations. If $\gamma=s_{1} s_{2} \ldots s_{n}$ is a reduced representation, it has $s_{1} \in\{a, b, c\}$ and for every $i \geq 2, s_{i} \in\{a, b, c\} \backslash\left\{s_{i-1}\right\}$. Therefore $\left|\Gamma_{n}\right|=3 \cdot 2^{n-1}$.

\subsection{Minkowski function and measure}

The Minkowski question mark function was first constructed by Hermann Minkowski and is studied in the field of Diophantine approximations. It is traditionally labeled by "?" but for readability purposes we label it through this paper by $\mathcal{M}$. If $\left[a_{0} ; a_{1}, a_{2}, \ldots, a_{n}\right]$ is the continued fraction representation of $x \in \mathbb{Q}$, then

$$
\mathcal{M}(x)=a_{0}+\sum_{k=1}^{n} \frac{(-1)^{k+1}}{2^{a_{1}+\cdots+a_{k}}} .
$$

If $x=\left[a_{0} ; a_{1}, a_{2} \ldots\right]$ is irrational, then the summation becomes infinite. We briefly describe an equivalent construction which will be more useful for our needs. 
$\mathcal{M}$ is first defined as a function from $\mathbb{Q} \cap[0,1]$ to the dyadic rationals $\mathbb{Q}_{2} \cap[0,1]$ and then extended to all of $[0,1]$ using continuity arguments. For more details see [1]. If $q$ is the $(k+1)$ th term in $\mathscr{F}_{n}$, that is,

$$
\mathscr{F}_{n}=\left(q_{1}, q_{2}, \ldots, q_{k}, q, q_{k+2}, \ldots, q_{m}\right) \text {, then } \mathcal{M}(q):=\frac{k}{2^{n}} .
$$

Notice that every $q \in \mathbb{Q}$ appears in infinitely many $\mathscr{F}_{n}$ but the definition does not depends on the choice of $n$. The next lemma will turn out useful in the next section.

Lemma 2.10. Let $p<q$ be a Farey pair in $\mathscr{F}_{n}$. Then $\mathcal{M}(q)-\mathcal{M}(p)=\frac{1}{2^{n}}$.

$\mathcal{M}$ is an ascending continuous bounded function, thus it is uniformly continuous. Since $\mathbb{Q}$ is dense in $[0,1]$ it can be extended to a continuous function from $[0,1]$ to $[0,1]$. This function can be used to construct a Lebesgue-Stieltjes measure on $[0,1]$ by defining

$$
\mu_{\mathcal{M}}([a, b))=\mathcal{M}(b)-\mathcal{M}(a)
$$

and extending using Carathéodory extension. Our purposes demand extending $\mu_{\mathcal{M}}$ to the entire real line. The following extension will turn out to be useful. For $q \in[n, n+1]$ define

$$
\overline{\mathcal{M}}(q)=\frac{1}{3}\left(\sum_{k=-\infty}^{n-1} \frac{1}{2^{|k|}}+\frac{\mathcal{M}(\{q\})}{2^{|n|}}\right),
$$

where $\{q\}$ denotes the fractional part of $q$. Using the same methods used for $\mathcal{M}$ we construct the extended Minkowski measure $\mu_{\overline{\mathcal{M}}}$. Notice that

and

$$
\lim _{q \rightarrow-\infty} \overline{\mathcal{M}}(q)=0
$$

$$
\lim _{q \rightarrow \infty} \overline{\mathcal{M}}(q)=1
$$

thus $\mu_{\mathcal{M}}$ is a probability measure.

Notice that the tight connection between the Farey tessellation and continued fractions is not new. Series has shown in [2] that the continued fraction expansion of any $x \in \mathbb{R}$ can be read from its position relative to the Farey tessellation. It follows from her work that if $T(p, q, s)$ is a tile with

then

$$
p, q, s<\infty \text { and } q=\left[a_{0} ; a_{1}, \ldots, a_{n}\right],
$$

$$
p \oplus q, \quad q \oplus s \in\left\{\left[a_{0} ; a_{1}, \ldots, a_{n}+1\right], \quad\left[a_{0} ; a_{1}, \ldots, a_{n}-1,2\right]\right\} .
$$

Therefore, for any tile $T(p, q, s)$ with finite vertices, if $p=\left[a_{0} ; a_{1}, \ldots, a_{n}\right]$ and $p \oplus q=\left[b_{0} ; b_{1}, \ldots, b_{m}\right]$, then $\sum_{i=0}^{m} b_{i}=1+\sum_{i=0}^{n} a_{i}$. This suggests an alternative approach for proving some claims presented here. 


\section{Main results}

\subsection{Word metric}

The group $G=\operatorname{PSL}(2, \mathbb{R})$ has a natural action on the projective line $X=$ $P\left(\mathbb{R}^{2}\right)$ which stems from the linear action on $\mathbb{R}^{2}$. For $g=\left[\left(\begin{array}{ll}a & b \\ c & d\end{array}\right)\right] \in G$ and $\left[\left(\begin{array}{l}x \\ y\end{array}\right)\right]=x \in X$ the $G$ action on $X$ is defined by

$$
g x=\left[\left(\begin{array}{c}
a x+b y \\
c x+d y
\end{array}\right)\right] .
$$

This action is well defined and does not depend of the choice of representatives in either $X$ or $G$. We identify $X$ with $\partial \mathbb{H}=\mathbb{R} \cup\{\infty\}$ using $\left[\left(\begin{array}{l}x \\ y\end{array}\right)\right] \rightarrow \frac{x}{y}$ when $y \neq 0$ and $\left[\left(\begin{array}{l}1 \\ 0\end{array}\right)\right] \rightarrow \infty$. $G$ acts on $\partial \mathbb{H}$ with Mobius transformations. By choosing suitable representatives we see that $\partial \mathbb{H}$ and $X$ are in fact isomorphic $G$-sets:

$$
g\left[\left(\begin{array}{l}
x \\
y
\end{array}\right)\right]=g\left[\left(\begin{array}{c}
\frac{x}{y} \\
1
\end{array}\right)\right]=g\left[\left(\begin{array}{c}
z \\
1
\end{array}\right)\right]=\left[\left(\begin{array}{c}
a z+b \\
c z+d
\end{array}\right)\right]=\left[\left(\begin{array}{c}
\frac{a z+b}{c z+d} \\
1
\end{array}\right)\right] .
$$

We first prove Theorem 1.1 for $z \in\{0,1, \infty\}$.

Lemma 3.1. For every $f \in C(X)$ and for $z \in\{0,1, \infty\}$ :

$$
\lim _{n \rightarrow \infty} \frac{S_{n}(f, z)}{\left|\Gamma_{n}\right|}=\int_{X} f \mathrm{~d} \mu_{\overline{\mathcal{M}}} .
$$

Proof. Let $f=\mathbb{1}_{[p, q]}$ with $p, q \in \mathbb{Q} \cap[0,1]$ a Farey pair. The summation $S_{n}(f, z)$ can be expressed as $S_{n}(f, z)=\left|\left\{\gamma \in \Gamma_{n}: \gamma z \in[p, q]\right\}\right|$. Since $p, q$ are a Farey pair they are vertices of some triangle $T(p, s, q) \in \mathscr{T}$. Let $g \in \Gamma$ such that $T(p, s, q)=\Delta_{g}$ and let $N:=\|g\|$. Let $\gamma \in \Gamma$ with $\Delta_{\gamma}=T(u, v, w)$. Since elements of $\Gamma$ move vertices of tiles to vertices of tiles, and since $\{0,1, \infty\}$ are the vertices of $\Delta_{e}, \gamma z$ is a vertex of $\Delta_{\gamma}$. Therefore $\gamma z \in[p, q]$ implies either $[u, w] \subset[p, q]$ or $w=p$ or $u=q$. The typical case is $[u, w] \subset[p, q]$ and for every $n$ exist at most 2 different $\gamma$ such that the other cases occur. Using Lemma 2.6 and a simple combinatorial argument we deduce that for every $n>N, S_{n}(f, z)=2^{n-N}+\theta(n)$ with $\theta(n) \in\{0,1,2\}$. Using Lemma 2.9 we get

$$
\lim _{n \rightarrow \infty} \frac{S_{n}(f, z)}{\left|\Gamma_{n}\right|}=\lim _{n \rightarrow \infty} \frac{2^{n-N}+\theta(n)}{3 \cdot 2^{n-1}}=\frac{1}{3} 2^{1-N} .
$$

Since $\|g\|=N$ and using Lemma 2.7 we see that $p, q$ are Farey pair in $\mathscr{F}_{N-1}$. Lemma 2.10 implies

and therefore,

$$
\mathcal{M}(q)-\mathcal{M}(p)=2^{1-N}
$$

$$
\lim _{n \rightarrow \infty} \frac{S_{n}(f, z)}{\left|\Gamma_{n}\right|}=\frac{1}{3}(\mathcal{M}(q)-\mathcal{M}(p))=\overline{\mathcal{M}}(q)-\overline{\mathcal{M}}(p) .
$$


If $p, q \in \mathbb{Q} \cap[m, m+1], m \in \mathbb{Z}$, we use similar arguments. This time to compute $S_{n}(f, z)=\left|\left\{\gamma \in \Gamma_{n}: \gamma z \in[p, q]\right\}\right|$ notice that $\gamma z \in[p, q]$ implies $\gamma z \in[m, m+1]$, therefore $\Delta_{\gamma}=T(u, v, w)$ has $[u, w] \subset[m, m+1]$ or $w=m$ or $u=m+1$. If the reduced representation of $\gamma$ is

it must have

$$
\gamma_{1} \ldots \gamma_{t} \text { and }[u, w] \subset[m, m+1]
$$

$$
\Delta_{\gamma_{1} \ldots \gamma_{|m|}}=T(m, m+1, \infty)
$$

Using same counting method as before we see

$$
\lim _{n \rightarrow \infty} \frac{S_{n}(f, z)}{\left|\Gamma_{n}\right|}=\frac{1}{3} \frac{1}{2^{|m|}}(\mathcal{M}(\{q\})-\mathcal{M}(\{p\}))=\overline{\mathcal{M}}(q)-\overline{\mathcal{M}}(p) .
$$

Now let $f \in C(X)$. Since $X$ is compact we can find a sequence of simple functions $f_{m}$ which converge uniformly to $f$. That is, there exists a sequence of simple functions $f_{m}=\sum c_{i} \mathbb{1}_{i}$ with $\mathbb{1}_{i}=\mathbb{1}_{\left[p_{i}, q_{i}\right]}$ indicator functions such that

$$
\epsilon(m):=\sup _{x \in X}\left|f_{m}(x)-f(x)\right| \text { has } \lim _{m \rightarrow \infty} \epsilon(m)=0 .
$$

Lemma 2.1 implies density of Farey pairs in $[0,1]$ so we can take $p_{i}, q_{i}$ to be integral translations of Farey pairs and get

$$
\begin{aligned}
& \lim _{n \rightarrow \infty}\left|\frac{S_{n}(f, z)}{\left|\Gamma_{n}\right|}-\int_{X} f \mathrm{~d} \mu_{\overline{\mathcal{M}}}\right| \\
& =\lim _{n \rightarrow \infty}\left|\frac{S_{n}(f, z)}{\left|\Gamma_{n}\right|}-\frac{S_{n}\left(f_{m}, z\right)}{\left|\Gamma_{n}\right|}+\frac{S_{n}\left(f_{m}, z\right)}{\left|\Gamma_{n}\right|}-\int_{X} f_{m} \mathrm{~d} \mu_{\overline{\mathcal{M}}}+\int_{X} f_{m} \mathrm{~d} \mu_{\overline{\mathcal{M}}}-\int_{X} f \mathrm{~d} \mu_{\overline{\mathcal{M}}}\right| \\
& \leq \lim _{n \rightarrow \infty}\left(\left|\frac{S_{n}\left(f-f_{m}, z\right)}{\left|\Gamma_{n}\right|}\right|+\left|\frac{S_{n}\left(f_{m}, z\right)}{\left|\Gamma_{n}\right|}-\int_{X} f_{m} \mathrm{~d} \mu_{\overline{\mathcal{M}}}\right|+\left|\int_{X}\left(f-f_{m}\right) \mathrm{d} \mu_{\overline{\mathcal{M}}}\right|\right) \\
& \leq \lim _{n \rightarrow \infty}\left(\epsilon(m)+\left|\frac{S_{n}\left(f_{m}, z\right)}{\left|\Gamma_{n}\right|}-\int_{X} f_{m} \mathrm{~d} \mu_{\overline{\mathcal{M}}}\right|+\epsilon(m)\right)=2 \epsilon(m) .
\end{aligned}
$$

We may now take $m \rightarrow \infty$ and get

$$
\begin{aligned}
\lim _{n \rightarrow \infty}\left|\frac{S_{n}(f, z)}{\left|\Gamma_{n}\right|}-\int_{X} f \mathrm{~d} \mu_{\mathcal{M}}\right| & =\lim _{m \rightarrow \infty} \lim _{n \rightarrow \infty}\left|\frac{S_{n}(f, z)}{\left|\Gamma_{n}\right|}-\int_{X} f \mathrm{~d} \mu_{\overline{\mathcal{M}}}\right| \\
& \leq \lim _{m \rightarrow \infty} 2 \epsilon(m)=0 .
\end{aligned}
$$


We are now ready to prove the main theorem.

Proof of Theorem 1.1. Let $f \in C(X)$ and $z \in X=\mathbb{R} \cup\{\infty\}$. We show

$$
\lim _{n \rightarrow \infty} \frac{1}{\left|\Gamma_{n}\right|} \sum_{\gamma \in \Gamma_{n}}|f(\gamma z)-f(\gamma 0)|=0
$$

We first divide $\Gamma_{n}$ into four disjoint sets. For $\delta, R, L>0$ define :

$$
\begin{aligned}
\Gamma_{n}^{\delta} & =\left\{\left[\left(\begin{array}{ll}
a & b \\
c & d
\end{array}\right)\right] \in \Gamma_{n}:\left|z+\frac{d}{c}\right|<\delta\right\}, \\
\Gamma_{n}^{\delta, R, L+} & =\left\{\left[\left(\begin{array}{ll}
a & b \\
c & d
\end{array}\right)\right] \in \Gamma_{n}: R>\left|z+\frac{d}{c}\right| \geq \delta,|c|<L\right\}, \\
\Gamma_{n}^{\delta, R, L-} & =\left\{\left[\left(\begin{array}{ll}
a & b \\
c & d
\end{array}\right)\right] \in \Gamma_{n}: R>\left|z+\frac{d}{c}\right| \geq \delta,|c| \geq L\right\}, \\
\Gamma_{n}^{R} & =\left\{\left[\left(\begin{array}{ll}
a & b \\
c & d
\end{array}\right)\right] \in \Gamma_{n}:\left|z+\frac{d}{c}\right| \geq R\right\},
\end{aligned}
$$

and denote $\Gamma_{n}^{\delta, R, L \pm}:=\Gamma_{n}^{\delta, R, L+} \cup \Gamma_{n}^{\delta, R, L-}$. These sets are well defined as both $\frac{d}{c}$ and $|c|$ are the same for $\pm\left(\begin{array}{ll}a & b \\ c & d\end{array}\right)$. Notice that $\gamma=\left[\left(\begin{array}{ll}a & b \\ c & d\end{array}\right)\right]$ has $\gamma \frac{-d}{c}=\infty$ hence $\gamma^{-1} \infty=\frac{-d}{c}$. Using Lemma 1.1 with $z=\infty$ and the fact that $\|\gamma\|=\left\|\gamma^{-1}\right\|$ we get

$$
\begin{aligned}
\lim _{n \rightarrow \infty} \frac{\left|\Gamma_{n}^{\delta}\right|}{\left|\Gamma_{n}\right|} & =\lim _{n \rightarrow \infty} \frac{\left|\left\{\gamma \in \Gamma_{n}:\left|z-\gamma^{-1} \infty\right|<\delta\right\}\right|}{\left|\Gamma_{n}\right|} \\
& =\lim _{n \rightarrow \infty} \frac{\left|\gamma \in \Gamma_{n}: \gamma^{-1} \infty \in[z-\delta, z+\delta]\right|}{\left|\Gamma_{n}\right|} \\
& =\lim _{n \rightarrow \infty} \frac{S_{n}\left(\mathbb{1}_{[z-\delta, z+\delta]}, \infty\right)}{\left|\Gamma_{n}\right|}=\mu_{\mathcal{M}}([z-\delta, z+\delta]) .
\end{aligned}
$$

We apply same reasoning for $\Gamma_{n}^{\delta}$ and get

$$
\lim _{n \rightarrow \infty} \frac{\left|\Gamma_{n}^{R}\right|}{\left|\Gamma_{n}\right|}=\mu_{\overline{\mathcal{M}}}([z+R, \infty) \cup[z-R,-\infty)) .
$$

$f$ is continuous on compact space, therefore bounded by some $B \in \mathbb{R}$ therefore,

$$
\begin{aligned}
\frac{1}{\left|\Gamma_{n}\right|} \sum_{\gamma \in \Gamma_{n}^{\delta}} \mid & f(\gamma z)-f(\gamma 0)\left|+\frac{1}{\left|\Gamma_{n}\right|} \sum_{\gamma \in \Gamma_{n}^{R}}\right| f(\gamma z)-f(\gamma 0) \mid \leq \\
& B\left(\mu_{\overline{\mathcal{M}}}([z-\delta, z+\delta])+\mu_{\overline{\mathcal{M}}}([z+R, \infty) \cup[z-R,-\infty))\right) \underset{\delta \rightarrow 0}{\longrightarrow} 0 .
\end{aligned}
$$

The convergence being due to continuity of $\overline{\mathcal{M}}$. To bound the sum over $\Gamma_{n}^{\delta, R, L \pm}$ we approximate $|\gamma z-\gamma 0|$. Let $\gamma=\left[\left(\begin{array}{ll}a & b \\ c & d\end{array}\right)\right] \in \Gamma_{n}^{\delta, R, L \pm}$ and assume $d \neq 0$. 
If $d=0$, then $\gamma 0=\infty$ and $\Delta_{\gamma}=T(m, m+1, \infty)$ with some $m \in \mathbb{Z}$. There are at most 2 such $\gamma$ in $\Gamma_{n}$. Notice that $\gamma \in \Gamma_{n}^{\delta, R, L \pm}$ implies $c z+d \neq 0$ so we can write

$$
|\gamma z-\gamma 0|=\left|\frac{a z+b}{c z+d}-\frac{b}{d}\right|=\left|\frac{z}{d c\left(z+\frac{d}{c}\right)}\right| \leq \frac{|z|}{\delta|c|} .
$$

Every $\gamma \in \Gamma_{n}^{\delta, R, L-}$ has $\gamma^{-1}=-\frac{d}{c} \in[z-R, z+R]$. Lemma 2.8 implies that for each $n$ there are at most 2 different $\gamma \in \Gamma_{n}$ that can have $\gamma_{1} \infty=\gamma_{2} \infty$. Since $\gamma^{-1} \infty \in \mathbb{Q}$ and $|c|<L$ we can bound

$$
\begin{aligned}
\left|\Gamma_{n}^{\delta, R, L-}\right| & \leq 2\left|\gamma^{-1} \infty: \gamma \in \Gamma_{n}^{\delta, R, L-}\right| \\
& \leq 2\left|\left\{\frac{p}{q} \in \mathbb{Q} \cap[z-R, z+R]: q<L\right\}\right| \\
& \leq 2(2 R+1)(L+(L-1)+\cdots+1)<2(2 R+1) L^{2} .
\end{aligned}
$$

If $\gamma \in \Gamma_{n}^{\delta, R, L+}$ it has $c \geq L$ hence $|\gamma z-\gamma 0| \leq \frac{|z|}{\delta L}$ and uniform continuity of $f$, then implies $|f(\gamma z)-f(\gamma 0)|<\epsilon(L) \underset{L \rightarrow \infty}{\longrightarrow} 0$. Putting everything together we get

$$
\begin{aligned}
& \lim _{n \rightarrow \infty}\left|\frac{S_{n}(f, z)}{\left|\Gamma_{n}\right|}-\frac{S_{n}(f, 0)}{\left|\Gamma_{n}\right|}\right| \leq \lim _{n \rightarrow \infty} \frac{1}{\left|\Gamma_{n}\right|} \sum_{\gamma \in \Gamma_{n}}|f(\gamma z)-f(\gamma 0)| \\
& =\lim _{n \rightarrow \infty} \frac{1}{\left|\Gamma_{n}\right|}\left(\sum_{\gamma \in \Gamma_{n}^{\delta}}|f(\gamma z)-f(\gamma 0)|+\sum_{\gamma \in \Gamma_{n}^{\delta, R, L \pm}}|f(\gamma z)-f(\gamma 0)|+\sum_{\gamma \in \Gamma_{n}^{R}}|f(\gamma z)-f(\gamma 0)|\right) \\
& \leq B \mu_{\overline{\mathcal{M}}}([z-\delta, z+\delta])+B \mu_{\overline{\mathcal{M}}}([z+R, \infty) \cup[z-R,-\infty))+\epsilon(L) .
\end{aligned}
$$

We can now take $L, R \rightarrow \infty$ and $\delta \rightarrow 0$ and get

$$
\begin{aligned}
& \lim _{n \rightarrow \infty}\left|\frac{S_{n}(f, z)}{\left|\Gamma_{n}\right|}-\frac{S_{n}(f, 0)}{\left|\Gamma_{n}\right|}\right|=\lim _{\delta \rightarrow 0} \lim _{R \rightarrow \infty} \lim _{L \rightarrow \infty} \lim _{n \rightarrow \infty}\left|\frac{S_{n}(f, z)}{\left|\Gamma_{n}\right|}-\frac{S_{n}(f, 0)}{\left|\Gamma_{n}\right|}\right| \\
& \leq \lim _{\delta \rightarrow 0} \lim _{R \rightarrow \infty} \lim _{L \rightarrow \infty}\left(B \mu_{\overline{\mathcal{M}}}([z-\delta, z+\delta])+B \mu_{\overline{\mathcal{M}}}([z+R, \infty) \cup[z-R,-\infty))+\epsilon(L)\right) \\
& =0 .
\end{aligned}
$$

\subsection{Stationary measure and random walk average}

We now prove Theorem 1.2, stating that the extended Minkowsi probability measure is, in fact, stationary with respect to the random wal defined by $\mu(a)=\mu(b)=\mu(c)=\frac{1}{3}$. Notice that by Furstenberg's uniqueness Theorem [3] the stationary measure in this case is unique. 
Pro of. Let $A \subset X$ be measurable. Then

$$
\begin{aligned}
\mu * \mu_{\overline{\mathcal{M}}}(A) & =\frac{1}{3}\left(\mu_{\overline{\mathcal{M}}}(a A)+\mu_{\overline{\mathcal{M}}}(b A)+\mu_{\overline{\mathcal{M}}}(c A)\right) \\
& =\frac{1}{3} \lim _{n \rightarrow \infty} \frac{1}{\left|\Gamma_{n}\right|}\left(S_{n}\left(\mathbb{1}_{a A}, 0\right)+S_{n}\left(\mathbb{1}_{b A}, 0\right)+S_{n}\left(\mathbb{1}_{c A}, 0\right)\right) \\
& =\frac{1}{3} \lim _{n \rightarrow \infty} \frac{1}{\left|\Gamma_{n}\right|} \sum_{\gamma \in \Gamma_{n}}\left(\mathbb{1}_{A}(a \gamma 0)+\mathbb{1}_{A}(b \gamma 0)+\mathbb{1}_{A}(c \gamma 0)\right) .
\end{aligned}
$$

Lemma 2.4 implies that this is equal to

$$
\begin{aligned}
& \frac{1}{3} \lim _{n \rightarrow \infty} \frac{1}{\left|\Gamma_{n}\right|}\left(S_{n+1}\left(\mathbb{1}_{A}, 0\right)+2 S_{n-1}\left(\mathbb{1}_{A}, 0\right)\right) \\
& =\lim _{n \rightarrow \infty} \frac{1}{3}\left(2 \frac{S_{n+1}\left(\mathbb{1}_{A}, 0\right)}{\left|\Gamma_{n+1}\right|}+\frac{S_{n-1}\left(\mathbb{1}_{A}, 0\right)}{\left|\Gamma_{n-1}\right|}\right)=\frac{1}{3}\left(2 \mu_{\overline{\mathcal{M}}}(A)+\mu_{\overline{\mathcal{M}}}(A)\right)=\mu_{\overline{\mathcal{M}}}(A)
\end{aligned}
$$

The fact that the stationary measure and the word metric limit coincide is somewhat surprising. The following theorem generalizes the conditions under this occurs.

Definition 3.1. Let $\mu$ be a probability measure on $G$ with $\operatorname{supp}(\mu)=S \subset G$ such that $S$ generates $G$. We denote $G_{n}=\left\{g \in G:\|g\|_{S}=n\right\}$.

(1) $\mu$ is called evenly distributed if the mass that $\mu^{* n}$ assigns to an element depends only on its word metric. That is, for any $n, m \in \mathbb{N}$ exists $1 \geq \mu_{n, m} \geq 0$ such that any $g \in G$ with $\|g\|_{S}=m$ has $\mu^{* n}(g)=\mu_{n, m}$.

(2) $G$ action on a compact space $X$ is said to converge in word metric with respect to $S$ if for any $f \in C(X)$ and for any $x \in X$ the following limit exists

$$
\lim _{n \rightarrow \infty} \frac{1}{\left|G_{n}\right|} \sum_{g \in G_{n}} f(g x) .
$$

Notice that using Riesz representation theorem we know that if $G$ converges in word metric, then it converges to a space average with respect to some measure $\nu$.

TheOREM 3.2. Let $G$ be a group acting continuously on compact space $X$. Let $\mu$ be a probability measure on $G$ such that $S:=\operatorname{supp}(\mu)$ generates $G$. Assume $\mu$ is evenly distributed and that $G$ action on $X$ converges in word metric with respect to $S$ to a space average with respect to $\nu$. Then $\nu$ is $\mu$ stationary.

To prove this theorem we make use of two lemmas. Denote by $\delta_{x}$ the Dirac measure at point $x$.

Lemma 3.3. In same settings as Theorem 3.2, for any $x \in X$

$$
\mu^{* n} * \delta_{x} \stackrel{\text { weak-* }}{\longrightarrow} \nu .
$$


WORD METRIC, STATIONARY MEASURE AND MINKOWSKI'S FUNCTION

Pr o of. Let $f \in C(X)$. Since $\mu$ is evenly distributed we can write

$$
\mu^{* n} * \delta_{x}(f)=\int_{G} f(\gamma x) \mathrm{d} \mu^{* n}(\gamma)=\sum_{m=1}^{n} \sum_{\gamma \in \Gamma_{m}} \mu^{* n}(\gamma) f(\gamma x)=\sum_{m=1}^{n} \mu_{n, m} S_{m}(f, x) .
$$

$G$ action on $X$ converges in word metric, hence we approximate

$$
S_{m}(f, x)=\left|G_{m}\right|(\nu(f)+\epsilon(m)) \text { with } \epsilon(m) \underset{m \rightarrow \infty}{\longrightarrow} 0 .
$$

$\mu^{* n}$ is a probability measure hence $\sum_{m=1}^{n} \mu_{n, m}\left|G_{m}\right|=1$ and therefore

$$
\sum_{m=1}^{n} \mu_{n, m}\left|G_{m}\right|(\nu(f)+\epsilon(m))=\nu(f)+\sum_{m=1}^{n} \mu_{n, m}\left|G_{m}\right| \epsilon(m) .
$$

Let $M=\sup (\{\epsilon(m): m \in \mathbb{N}\})$ and $k \in \mathbb{N}$. The second term can be bounded by

$$
\sum_{m=1}^{n} \mu_{n, m}\left|G_{m}\right| \epsilon(m) \leq \mu^{* n}\left(\bigcup_{i \leq k} G_{i}\right) M+\max (\{\epsilon(m): m>k\}) .
$$

Since for any $k, \lim _{n \rightarrow \infty} \mu^{* n}\left(\bigcup_{i \leq k} G_{i}\right)=0$ we get

$$
\lim _{n \rightarrow \infty} \mu^{* n} * \delta_{x}(f) \leq \nu(f)+\max (\{\epsilon(m): m>k\})
$$

$k$ is arbitrary hence we are done.

LEMma 3.4. Let $G$ be a group acting continuously on $X$. Let $\pi$ be a probability measure on $X$ and $\mu$ a probability measure on $G$. Assuming

$$
\mu^{* n} * \pi \stackrel{\text { weak-* }}{\longrightarrow} \nu \quad \text { implies that } \nu \text { is } \mu \text { stationary. }
$$

P r o o f. let $\nu_{n}:=\mu^{* n} * \pi$. Since the space of measures is metric and since $\nu_{n}$ converges to $\nu$, the Cesaro average $\frac{1}{n} \sum_{k=1}^{n} \nu_{k}$ converges to $\nu$ as well. The difference measure $\Delta_{n}:=\nu-\nu_{n}$ has $\lim _{n \rightarrow \infty} \Delta_{n}=0$. Then

$$
\begin{aligned}
|\mu * \nu-\nu| & =\left|\mu *\left(\frac{1}{n} \sum_{k=1}^{n} \mu^{* k} * \pi+\Delta_{n}\right)-\left(\frac{1}{n} \sum_{k=1}^{n} \mu^{* k} * \pi+\Delta_{n}\right)\right| \\
& =\left|\frac{\nu_{n+1}-\nu_{1}}{n}+\mu * \Delta_{n}-\Delta_{n}\right| .
\end{aligned}
$$

Since $\nu_{k}$ are probability measures for all $k$ and since $\mu * \Delta_{n}$ tends to 0 we get

$$
|\mu * \nu-\nu|=\lim _{n \rightarrow \infty}|\mu * \nu-\nu|=\lim _{n \rightarrow \infty}\left|\frac{\nu_{n+1}-\nu_{1}}{n}+\mu * \Delta_{n}-\Delta_{n}\right|=0 .
$$

The fact that the random walk converges both to a stationary measure and the word metric limit proves Theorem 3.2 . One can check that the conditions of Theorem 3.2 apply to the Farey group acting on the projective line thus Theorem 1.3 follows as well. 


\section{URIYA PUMERANTZ}

\section{REFERENCES}

[1] PELEGRÍ, V.-PARAdÍS, J.-BIBILONI, L.: A new light on Minkowski's ?(x) function, J. Number Theory 73 (1998), no. 2, 212-227.

[2] SERIES, C.: The modular surface and continued fractions, J. London Math. Soc. (2) 31 (1985), no. 1, 69-80.

[3] BENOIST, Y.- QUINT, J.-F.: Random Walks on Reductive Groups. In: Ergebnisse der Mathematik und ihrer Grenzgebiete. 3. Folge. A Series of Modern Surveys in Mathematics [Results in Mathematics and Related Areas. 3rd Series. A Series of Modern Surveys in Mathematics] Vol. 62, Springer, Cham, 2016.

[4] BJORNER, A.-BRENTI, F.: Combinatorics of Coxeter Groups. In: Grad. Texts in Math. Vol. 231, Springer-Verlag, Berlin, New York, NY, 2005.

[5] BEARDON, A. F.: The Geometry of Discrete Groups. In: Graduate Texts in Mathematics Vol. 91, Springer-Verlag, Berlin, New York, Ny, 1st ed. 1983; Corr. 2nd printing 1995 Edition

[6] MASKIT, B.: On Poincaré's theorem for fundamental polygons, Advances in Math. 7 (1971), 219-230.

[7] LEDRAPPIER, F.: Distribution des orbites des réseaux sur le plan réel, C. R. Acad. Sci. Paris Sér. I Math. [Distribution of lattice orbits on the real plane] 329 (1999), no. 1, $61-64$.

[8] NOGUEIRA, A.: Orbit distribution on $\mathbb{R}^{2}$ under the natural action of $S L(2, \mathbb{Z})$, Indag. Math., N.S. 13 (2002), no. 1, 103-124.

[9] GORODNIK, A.-WEISS, B.: Distribution of lattice orbits on homogeneous varieties, Geom. Funct. Anal. 17 (2007), no. 1, 58-115.

[10] MAUCOURANT, F.-SCHAPIRA, B.: Distribution of orbits in $\mathbb{R}^{2}$ of a finitely generated group of $S L(2, \mathbb{R})$, Amer. J. Math. 136 (2014), no. 6, 1497-1542.

Received June 20, 2020

Accepted July 14, 2020

\section{Uriya Pumerantz \\ Department of Mathematics \\ Tel Aviv University 69978 Tel-Aviv, ISRAEL}

E-mail: pumerantz.uriya@gmail.com 